\title{
Conditions for a bigraph to be super-cyclic
}

\author{
Alexandr Kostochka* \\ University of Illinois at Urbana-Champaign \\ Urbana, Illinois, U.S.A. \\ Sobolev Institute of Mathematics \\ Novosibirsk, Russia \\ kostochk@math.uiuc.edu
}

\author{
Mikhail Lavrov \\ Kennesaw State University \\ Marietta, Georgia, U.S.A. \\ mlavrov@kennesaw.edu
}

\author{
Ruth $\mathrm{LuO}^{\dagger}$ \\ University of California, San Diego \\ La Jolla, California, U.S.A. \\ ruluo@ucsd.edu
}

\author{
Dara Zirlin \\ University of Illinois at Urbana-Champaign \\ Urbana, Illinois, U.S.A. \\ zirlin2@illinois.edu
}

Submitted: Jul 1, 2020; Accepted: Nov 8, 2020; Published: Jan 15, 2021

(C) The authors. Released under the CC BY-ND license (International 4.0).

\begin{abstract}
A hypergraph $\mathcal{H}$ is super-pancyclic if for each $A \subseteq V(\mathcal{H})$ with $|A| \geqslant 3, \mathcal{H}$ contains a Berge cycle with base vertex set $A$. We present two natural necessary conditions for a hypergraph to be super-pancyclic, and show that in several classes of hypergraphs these necessary conditions are also sufficient. In particular, they are sufficient for every hypergraph $\mathcal{H}$ with $\delta(\mathcal{H}) \geqslant \max \left\{|V(\mathcal{H})|, \frac{|E(\mathcal{H})|+10}{4}\right\}$.

We also consider super-cyclic bipartite graphs: $(X, Y)$-bigraphs $G$ such that for each $A \subseteq X$ with $|A| \geqslant 3, G$ has a cycle $C_{A}$ such that $V\left(C_{A}\right) \cap X=A$. Super-cyclic graphs are incidence graphs of super-pancyclic hypergraphs, and our proofs use the language of such graphs.
\end{abstract}

Mathematics Subject Classifications: 05C35,05C38,05C65,05D05

${ }^{*}$ Research is supported in part by NSF grant DMS-1600592 and grants 18-01-00353A and 19-01-00682 of the Russian Foundation for Basic Research.

${ }^{\dagger}$ Research is supported in part by NSF grant DMS-1902808.

${ }^{\ddagger}$ Research is supported in part by Arnold O. Beckman Research Award (UIUC) RB20003. 


\section{Introduction}

\subsection{Longest cycles in bipartite graphs and hypergraphs}

For positive integers $n, m$, and $\delta$ with $\delta \leqslant m$, let $\mathcal{G}(n, m, \delta)$ denote the set of all bipartite graphs with a bipartition $(X, Y)$ such that $|X|=n \geqslant 2,|Y|=m$ and for every $x \in X$, $d(x) \geqslant \delta$. In 1981, Jackson [4] proved that if $\delta \geqslant \max \left\{n, \frac{m+2}{2}\right\}$, then every graph $G \in$ $\mathcal{G}(n, m, \delta)$ contains a cycle of length $2 n$, i.e., a cycle that contains all vertices of $X$. This result is sharp. Jackson also conjectured that if $G \in \mathcal{G}(n, m, \delta)$ is 2-connected, then the lower bound on $\delta$ can be weakened.

Conjecture 1 (Jackson [4]). Let $m, n, \delta$ be integers. If $\delta \geqslant \max \left\{n, \frac{m+5}{3}\right\}$, then every 2-connected graph $G \in \mathcal{G}(n, m, \delta)$ contains a cycle of length $2 n$.

Recently, the conjecture was proved in [8]. The restriction $\delta \geqslant \frac{m+5}{3}$ cannot be weakened any further because of the following example:

Example 2. Let $n_{1} \geqslant n_{2} \geqslant n_{3} \geqslant 1$ be such that $n_{1}+n_{2}+n_{3}=n$. Let $G_{3}\left(n_{1}, n_{2}, n_{3}\right) \in$ $\mathcal{G}(n, 3 \delta-4, \delta)$ be the bipartite graph obtained from $K_{\delta-2, n_{1}} \cup K_{\delta-2, n_{2}} \cup K_{\delta-2, n_{3}}$ by adding two vertices $a$ and $b$ that are both adjacent to every vertex in the parts of size $n_{1}, n_{2}$, and $n_{3}$. Then a longest cycle in $G_{3}\left(n_{1}, n_{2}, n_{3}\right)$ has length $2\left(n_{1}+n_{2}\right) \leqslant 2(n-1)$.

Very recently [9], the bound was refined for 3-connected graphs in $G \in \mathcal{G}(n, m, \delta)$.

Theorem 3 ([9]). Let $m, n, \delta$ be integers. If $\delta \geqslant \max \left\{n, \frac{m+10}{4}\right\}$, then every 3 -connected graph $G \in \mathcal{G}(n, m, \delta)$ contains a cycle of length $2 n$.

A construction very similar to Construction 2 shows that the bound $\frac{m+10}{4}$ is sharp.

The results can be translated into the language of hypergraphs and hamiltonian Berge cycles.

Recall that a hypergraph $\mathcal{H}$ is a set of vertices $V(\mathcal{H})$ and a set of edges $E(\mathcal{H})$ such that each edge is a subset of $V(\mathcal{H})$. We consider hypergraphs with edges of any size. The degree, $d(v)$, of a vertex $v$ is the number of edges that contain $v$.

A Berge cycle of length $\ell$ in a hypergraph is a set of $\ell$ distinct vertices $\left\{v_{1}, \ldots, v_{\ell}\right\}$ and $\ell$ distinct edges $\left\{e_{1}, \ldots, e_{\ell}\right\}$ such that for every $i \in[\ell], v_{i}, v_{i+1} \in e_{i}$ (indices are taken modulo $\ell$ ). The vertices $v_{1}, \ldots, v_{\ell}$ are the base vertices of the cycle.

Naturally, a hamiltonian Berge cycle in a hypergraph $\mathcal{H}$ is a Berge cycle whose set of base vertices is $V(\mathcal{H})$.

Let $\mathcal{H}=(V(\mathcal{H}), E(\mathcal{H}))$ be a hypergraph. The incidence graph of $\mathcal{H}$ is the bipartite graph $I(\mathcal{H})$ with parts $(X, Y)$ where $X=V(\mathcal{H}), Y=E(\mathcal{H})$ such that for $e \in Y, v \in X$, $e v \in E(I(\mathcal{H}))$ if and only if the vertex $v$ is contained in the edge $e$ in $\mathcal{H}$.

If $\mathcal{H}$ has $n$ vertices, $m$ edges and minimum degree at least $\delta$, then $I(\mathcal{H}) \in \mathcal{G}(n, m, \delta)$. There is a simple relation between the cycle lengths in a hypergraph $\mathcal{H}$ and its incidence graph $I(\mathcal{H})$ : If $\left\{v_{1}, \ldots, v_{\ell}\right\}$ and $\left\{e_{1}, \ldots, e_{\ell}\right\}$ form a Berge cycle of length $\ell$ in $\mathcal{H}$, then $v_{1} e_{1} \ldots v_{\ell} e_{\ell} v_{1}$ is a cycle of length $2 \ell$ in $I(\mathcal{H})$, and vice versa. 


\subsection{Super-pancyclic hypergraphs and super-cyclic bigraphs}

Recall that an $n$-vertex graph is pancyclic if it contains a cycle of length $\ell$ for every $3 \leqslant \ell \leqslant n$. There are a number of interesting results on pancyclic graphs, see e.g. survey [10]. A similar notion for hypergraphs and a strengthening of it were recently considered in [8].

A hypergraph $\mathcal{H}$ is pancyclic if it contains a Berge cycle of length $\ell$ for every $\ell \geqslant 3$. Furthermore, $\mathcal{H}$ is super-pancyclic if for every $A \subseteq V(\mathcal{H})$ with $|A| \geqslant 3, \mathcal{H}$ has a Berge cycle whose set of base vertices is $A$.

While the notion of super-pancyclic graphs is useless, since only complete graphs have this property, the notion for general hypergraphs is nontrivial. For example, Jackson's proof [4] that for $\delta \geqslant \max \left\{n, \frac{m+2}{2}\right\}$, each graph $G \in \mathcal{G}(n, m, \delta)$ has a cycle of length $2 n$ yields a stronger statement. In the language of hypergraphs, it implies the following.

Theorem 4 ([4]). If $\delta \geqslant \max \left\{n, \frac{m+2}{2}\right\}$, then every $n$-vertex hypergraph with $m$ edges and minimum degree at least $\delta$ is super-pancyclic.

It is interesting to find broader conditions guaranteeing that a hypergraph is superpancyclic. The notion of super-pancyclicity translates into the language of bipartite graphs as follows.

By an $(X, Y)$-bigraph we mean a bipartite graph $G$ with a specified ordered bipartition $(X, Y)$. An $(X, Y)$-bigraph is super-cyclic if for every $X^{\prime} \subseteq X$ with $\left|X^{\prime}\right| \geqslant 3, G$ has a cycle $C$ with $V(C) \cap X=X^{\prime}$; we say that $C$ is based on $X^{\prime}$.

To state necessary conditions for an $(X, Y)$-bigraph to be super-cyclic, we need a new notion. For $A \subseteq X$, the super-neighborhood $\widehat{N}(A)$ is the set $\{y \in Y:|N(y) \cap A| \geqslant 2\}$.

If $G$ is a super-cyclic $(X, Y)$-bigraph, $A \subseteq X$, and $C$ is a cycle based on $A$, let $B=V(C) \cap Y$. Then $B \subseteq \widehat{N}(A)$ and $G[A \cup B]$ is 2-connected. Since adding a vertex of degree at least 2 to a 2-connected graph keeps the graph 2-connected, we conclude that every super-cyclic bigraph satisfies the following ${ }^{1}$.

$$
\text { For each } A \subseteq X \text { with }|A| \geqslant 3:\left\{\begin{array}{l}
|\widehat{N}(A)| \geqslant|A|, \text { and } \\
G[A \cup \widehat{N}(A)] \text { is 2-connected. }
\end{array}\right.
$$

We conjecture that these necessary conditions for a bigraph to be super-cyclic are also sufficient.

Conjecture 5. If $G$ is an (X, $Y)$-bigraph satisfying (1), then $G$ is super-cyclic.

To give partial support for Conjecture 5, let us somewhat refine the notion of supercyclic bigraphs.

\footnotetext{
${ }^{1}$ Jaehoon Kim [6] observed that to check condition (1), it is sufficient to verify that $G[A \cup \widehat{N}(A)]$ is 2-connected only when $|A|=3$, though $|\widehat{N}(A)| \geqslant|A|$ still needs to be checked for all $A$. When $|A|>3$, if $G[A \cup \widehat{N}(A)]$ is not 2-connected, there is a subset $A^{\prime} \subseteq A$ with $\left|A^{\prime}\right|=3$ for which $G\left[A^{\prime} \cup \widehat{N}\left(A^{\prime}\right)\right]$ is also not 2-connected.
} 
For an integer $k \geqslant 3$, a bipartite graph $G$ with partition $(X, Y)$ is $k$-cyclic if for every $X^{\prime} \subseteq X$ with $\left|X^{\prime}\right|=k, G$ has a cycle $C$ that is based on $X^{\prime}$. If $G$ is $k$-cyclic for all $3 \leqslant k \leqslant|X|$, then it is super-cyclic.

In a series of claims, we prove the following.

Theorem 6. If $G$ is an (X,Y)-bigraph satisfying (1), then $G$ is $k$-cyclic for $k=3,4,5,6$.

Another result supporting Conjecture 5 was proved in [8] (in slightly different terms).

Theorem 7 ([8]). Let $\delta \geqslant \max \left\{n, \frac{m+5}{3}\right\}$. If $G \in \mathcal{G}(n, m, \delta)$ satisfies (1), then $G$ is super-cyclic.

We use Theorem 6 and the ideas of the proof of Theorem 3 to strengthen Theorem 7 as follows.

Theorem 8. Let $\delta \geqslant \max \left\{n, \frac{m+10}{4}\right\}$. If $G \in \mathcal{G}(n, m, \delta)$ satisfies (1), then $G$ is supercyclic.

In terms of hypergraphs, our result is as follows.

Corollary 9 (Hypergraph version of Theorem 8). Let $\delta \geqslant \max \left\{n, \frac{m+10}{4}\right\}$. If the incidence graph of an $n$-vertex hypergraph $\mathcal{H}$ with $m$ edges and minimum degree $\delta(\mathcal{H})$ satisfies $(1)$, then $\mathcal{H}$ is super-pancyclic.

We present the main proofs in the language of bipartite graphs. We will say that an $(X, Y)$-bigraph $G$ is critical if the following conditions hold:

(a) $G$ satisfies (1) but is not super-cyclic,

(b) $\widehat{N}(X)=Y$, and

(c) for every $X^{\prime} \subset X$ with $X^{\prime} \neq X, G\left[X^{\prime} \cup Y\right]$ is super-cyclic.

Note that every graph satisfying (1) is either super-cyclic or has a critical subgraph.

Furthermore, we say that a critical $(X, Y)$-bigraph $G$ is saturated if, after adding any $X, Y$-edge to $G$, the resulting graph is super-cyclic.

In Section 2 we prove basic properties of critical bigraphs. Based on this, in Section 3 we prove Theorem 6 for $k=3,4$, and 5. In Section 4 we discuss saturated critical graphs, which will be useful in the last two sections. In Section 5 we prove Theorem 6 for $k=6$. In Section 6 we prove Theorem 8.

\section{Properties of critical bigraphs}

For all $(X, Y)$-bigraphs $G$ below we assume $|X| \geqslant 3$, since $G$ is trivially super-cyclic when $|X| \leqslant 2$.

Lemma 10. Suppose that an $(X, Y)$-bigraph $G$ satisfies (1). Then $\left|N(x) \cap N\left(x^{\prime}\right)\right| \geqslant 1$ for all distinct $x, x^{\prime} \in X$. 
Proof. Let $x^{\prime \prime}$ be any vertex in $X-\left\{x, x^{\prime}\right\}$ and $A=\left\{x, x^{\prime}, x^{\prime \prime}\right\}$. If $N(x) \cap N\left(x^{\prime}\right)=\varnothing$, then $G[A \cup \widehat{N}(A)]-x^{\prime \prime}$ has no $x, x^{\prime}$-path, contradicting (1).

Claim 11. Let $G$ be a critical $(X, Y)$-bigraph. Then $G$ is 2-connected.

Proof. This is by the fact that $Y=\widehat{N}(X)$ and by (1).

Recall that for a vertex $v \in V(G)$ and a set $U \subseteq V(G)$, a $v, U$-fan of size $t$ is a set of $t$ paths from $v$ to $U$ such that the only common vertex of any two distinct paths is $v$. In view of Claim 11, the classical Dirac's Fan Lemma [3, 11] implies the following fact.

Lemma 12. Let $G$ be a critical $(X, Y)$-bigraph, $v \in V(G)$, and $U \subseteq V(G)$ with $|U| \geqslant 2$. Then $G$ has 2 paths from $v$ to $U$ having only the vertex $v$ in common.

Let $G$ be a critical $(X, Y)$-bigraph with $|X|=k+1$ and $x_{0} \in X$, where $k \geqslant 2$. Note that if $k<2$, then $G$ cannot be critical, since it is trivially super-cyclic. By definition, $G-\left\{x_{0}\right\}$ is super-cyclic. In particular, it has a cycle $C=x_{1} y_{1} x_{2} y_{2} \ldots x_{k} y_{k} x_{1}$ based on $X-\left\{x_{0}\right\}$. We index the vertices of $C$ modulo $k$; for example, $x_{k+1}=x_{1}$. We derive some properties of such triples $\left(G, x_{0}, C\right)$.

Claim 13. For all $y_{i}, y_{j} \in N\left(x_{0}\right), x_{i}$ and $x_{j}$ have no common neighbor outside $C$. Similarly, $x_{i+1}$ and $x_{j+1}$ have no common neighbor outside $C$.

Proof. If $x_{i}$ and $x_{j}$ have a common neighbor $y \notin V(C)$, then the cycle

$$
x_{1} y_{1} \ldots x_{i} y x_{j} y_{j-1} \ldots y_{i} x_{0} y_{j} x_{j+1} \ldots x_{1}
$$

is based on $X$, contrary to assumption. If $x_{i+1}$ and $x_{j+1}$ have such a common neighbor, consider the cycle $C$ in reverse and apply the same argument.

Claim 14. For every $y_{i} \in N\left(x_{0}\right), x_{i}$ and $x_{0}$ have no common neighbor outside $C$; similarly, $x_{i+1}$ and $x_{0}$ have no common neighbor outside $C$.

Proof. If $x_{i}$ and $x_{0}$ have a common neighbor $y \notin V(C)$, then we may extend $C$ to a cycle based on $X$ by replacing the edge $x_{i} y_{i}$ with the path $x_{i} y x_{0} y_{i}$. The proof for $x_{i+1}$ is similar.

Claim 15. For every $i$, if $x_{i}$ has a common neighbor $y$ with $x_{0}$ outside $C$, then $x_{i+1}$ has no common neighbor with $x_{0}$ outside $C$, except possibly for $y$.

Proof. If $x_{i+1}$ and $x_{0}$ have a common neighbor $y^{\prime} \notin V(C)$, with $y^{\prime} \neq y$, then we may extend $C$ to a cycle based on $X$ by replacing the path $x_{i} y_{i} x_{i+1}$ with the path $x_{i} y x_{0} y^{\prime} x_{i+1}$.

Lemma 16. The vertex $x_{0}$ has at least two neighbors in $C$. 
Proof. Let $A$ be the subset of $X$ consisting of $x_{0}$, together with all $x_{i}$ that do not have a common neighbor with $x_{0}$ outside $C$.

If $|A| \geqslant 3$, then $G[A \cup \widehat{N}(A)]$ is 2-connected by (1), so $x_{0}$ has at least two neighbors in $\widehat{N}(A)$. Each of these neighbors must also be adjacent to at least one vertex in $A-\left\{x_{0}\right\}$. By our choice of $A$, these neighbors must be in $C$, and we are done.

If $|A| \leqslant 2$, then $x_{0}$ has a common neighbor outside $C$ with all but at most one of $x_{1}, x_{2}, \ldots, x_{k}$. By Claim 15, two consecutive vertices $x_{i}, x_{i+1}$ cannot have different common neighbors with $x_{0}$ outside $C$. Therefore there is a vertex $y_{0}$ outside $C$ adjacent to $x_{0}$ and to all but at most one of $x_{1}, x_{2}, \ldots, x_{k}$.

By Claim 11, $d\left(x_{0}\right) \geqslant 2$. So there are two possibilities:

- If $x_{0}$ has a neighbor $y_{i}$ in $C$, then at least one of $x_{i}$ or $x_{i+1}$ is adjacent to $y_{0}$; then it has a common neighbor with $x_{0}$ outside $C$, contradicting Claim 14 .

- If $x_{0}$ has a neighbor $y_{0}^{\prime}$ outside $C$, then $y_{0}^{\prime}$ has a neighbor $x_{i}$ in $C$ because $\delta(G) \geqslant 2$. By Claim 15, $x_{i-1}$ and $x_{i+1}$ cannot have common neighbors with $x_{0}$ outside $C$ except possibly for $y_{0}^{\prime}$. If $x_{i-1} \neq x_{i+1}$ then at least one of them is adjacent to $y_{0}$, which is a contradiction. Otherwise, if $x_{i-1}=x_{i+1}$ then $k=2$ and $|X|=3$. If $x_{0}$ has a neighbor $y_{0}^{\prime \prime}$ that is adjacent to $x_{i-1}$, then we have a contradiction. If there is no such neighbor, then let $A=\left\{x_{0}, x_{i}, x_{i-1}\right\}$. We see that $G[A \cup \widehat{N}(A)]$ is not 2-connected, a contradiction.

Therefore the case $|A| \leqslant 2$ is impossible, completing the proof.

\section{3-, 4-, and 5-cyclic graphs}

Theorem 6 makes four claims: for $k=3,4,5,6$. In this section, we prove three of them.

Claim 17. All (X,Y)-bigraphs $G$ satisfying (1) are 3-cyclic.

Proof. Suppose the claim is false and take a vertex-minimal counter-example, so that $|X|=3$ and $Y=\widehat{N}(X)$. Then $G$ is critical. By Claim 11, $G$ is 2-connected, so it contains a cycle.

Suppose the longest cycle $C=x_{1} y_{1} x_{2} y_{2} x_{1}$ of $G$ has 4 vertices and does not include the vertex $x_{3}$. By Lemma 12, there are 2 paths from $x_{3}$ to $V(C)$ having only $x_{3}$ in common. Then $G$ would contain a cycle of length 6 unless the paths are just $x_{3} y_{1}$ and $x_{3} y_{2}$. Suppose that $y_{3} \in Y$ (note that $|Y| \geqslant 3$ by (1)). Again, by Lemma 12, there are 2 paths from $y_{3}$ to $V(C)$ having only $y_{3}$ in common. Then $G$ would contain a cycle of length 6 unless the paths are just $y_{3} x_{1}$ and $y_{3} x_{2}$. Then we get a 6-cycle $x_{1} y_{1} x_{3} y_{2} x_{2} y_{3} x_{1}$.

Claim 18. All (X,Y)-bigraphs $G$ satisfying (1) are 4-cyclic.

Proof. Suppose the claim is false and take a vertex-minimal counter-example, so that $|X|=4$ and $Y=\widehat{N}(X)$. Then $G$ is critical. Let $x_{0} \in X=\left\{x_{0}, x_{1}, x_{2}, x_{3}\right\}$ have maximum degree. By Claim 17, $G-\left\{x_{0}\right\}$ has a 6 -cycle $C=x_{1} y_{1} x_{2} y_{2} x_{3} y_{3} x_{1}$. 
Case 1: $x_{0}$ has a neighbor $y_{0}$ outside of $C$. By Lemma 16, $x_{0}$ is adjacent to at least two of $\left\{y_{1}, y_{2}, y_{3}\right\}$; since $\delta(G) \geqslant 2, y_{0}$ is adjacent to at least one of $\left\{x_{1}, x_{2}, x_{3}\right\}$. Then there is an edge of $C$ both of whose endpoints are adjacent to $x_{0}$ or $y_{0}$; without loss of generality, it's $x_{1} y_{1}$. We can replace $x_{1} y_{1}$ by $x_{1} y_{0} x_{0} y_{1}$, extending $C$.

Case 2: All of $x_{0}$ 's neighbors are in $C$. Then the neighbors of $x$ in $C$ have degree at least 3, and all other vertices in $Y$ at least 2. Since $|Y| \geqslant|X|$, there are also vertices of $X$ with degree 3 , and since $x_{0}$ was chosen to have maximum degree in $X$, its degree is at least 3. Therefore $x_{0}$ is adjacent to all of $\left\{y_{1}, y_{2}, y_{3}\right\}$.

Since $|Y| \geqslant 4$, there is $y_{0} \in Y$ outside $C$. By Claim 11, $y_{0}$ has at least two neighbors in $X$, and neither of them is $x_{0}$. Without loss of generality, $y_{0}$ is adjacent to $x_{1}$ and $x_{2}$, and so $G$ has a cycle $x_{0} y_{1} x_{2} y_{0} x_{1} y_{3} x_{3} y_{2} x_{0}$.

In both cases, we get an 8-cycle, a contradiction.

Claim 19. All (X,Y)-bigraphs $G$ satisfying (1) are 5-cyclic.

Proof. Suppose the claim is false and take a vertex-minimal counter-example, so that $|X|=5$ and $Y=\widehat{N}(X)$. Then $G$ is critical. Let $x_{0} \in X=\left\{x_{0}, x_{1}, x_{2}, x_{3}, x_{4}\right\}$ have maximum degree. By Claim 18, $G-\left\{x_{0}\right\}$ has an 8-cycle $C=x_{1} y_{1} x_{2} y_{2} x_{3} y_{3} x_{4} y_{4}$.

Case 1: $x_{0}$ has a neighbor $y_{0}$ outside of $C$. By Lemma 16, $x_{0}$ is adjacent to at least two of $\left\{y_{1}, y_{2}, y_{3}, y_{4}\right\}$; since $\delta(G) \geqslant 2, y_{0}$ is adjacent to at least one of $\left\{x_{1}, x_{2}, x_{3}, x_{4}\right\}$. In almost all cases, there is an edge of $C$ both of whose endpoints are adjacent to $x_{0}$ or $y_{0}$, in which case we are done as before. The remaining case is unique up to relabeling $C$; without loss of generality, $x_{0}$ is adjacent to $y_{1}$ and $y_{2}$ and $y_{0}$ is adjacent to $x_{4}$.

If $x_{3} y_{4}$ is an edge, then there is a 10-cycle $x_{1} y_{1} x_{2} y_{2} x_{0} y_{0} x_{4} y_{3} x_{3} y_{4} x_{1}$, and similarly there is a 10-cycle if $x_{1} y_{3}$ is an edge. If neither is an edge, then $\widehat{N}\left(\left\{x_{0}, x_{1}, x_{3}\right\}\right)$ contains $y_{1}$ and $y_{2}$, but not $y_{3}$ or $y_{4}$, so it needs a third vertex (call it $y_{5}$ ) which is outside $C$, adjacent to $x_{1}$ and either to $x_{0}$ or to $x_{3}$. In either case, we get a 10-cycle: one of

$$
x_{1} y_{5} x_{3} y_{2} x_{2} y_{1} x_{0} y_{0} x_{4} y_{4} x_{1} \text { or } x_{1} y_{5} x_{0} y_{1} x_{2} y_{2} x_{3} y_{3} x_{4} y_{4} x_{1} \text {. }
$$

Note that in Case 1, we did not use that $x_{0}$ has maximum degree.

Case 2: All of $x_{0}$ 's neighbors are in $C$. In this case, as before, we argue that $x_{0}$ must have degree at least 3 . Say $x_{0}$ is adjacent to $\left\{y_{1}, y_{2}, y_{3}\right\}$; we make no assumption about whether $x_{0}$ is adjacent to $y_{4}$.

We can replace $x_{2}$ or $x_{3}$ by $x_{0}$ to get new cycles using the same vertices $y_{1}, y_{2}, y_{3}, y_{4}$ of $Y$. If $x_{2}$ or $x_{3}$ has a neighbor other than $y_{1}, y_{2}, y_{3}, y_{4}$, then we can apply Case 1 .

So all the other vertices of $Y$ (and there must be at least one) must be adjacent only to $x_{1}$ and $x_{4}$. Since they can be swapped in for $y_{4}$ to get a new cycle, if $y_{4}$ is adjacent to any of $x_{0}, x_{2}, x_{3}$, we can also reduce to a cycle $C$ where Case 1 applies. Therefore $y_{4}$ is also adjacent only to $x_{1}$ and $x_{4}$.

But now $\widehat{N}\left(\left\{x_{0}, x_{1}, x_{2}, x_{3}\right\}\right)=\left\{y_{1}, y_{2}, y_{3}\right\}$ which violates (1). In all cases, we get a contradiction. 


\section{Saturated critical bigraphs}

Recall that a critical $(X, Y)$-bigraph $G$ is saturated if adding to $G$ any $X, Y$-edge results in a super-cyclic bigraph.

Lemma 20. If $G$ is a saturated critical $(X, Y)$-bigraph, then for every $y \in Y,|N(y)| \neq$ $|X|-1$.

Proof. Suppose $G$ is a saturated critical $(X, Y)$-bigraph, and for $y_{0} \in Y$ and $x_{0} \in X$ we have $N\left(y_{0}\right)=X-\left\{x_{0}\right\}$. Since $G$ is critical, $G-\left\{x_{0}\right\}$ is super-cyclic, but $G$ has no cycles based on $X$. Let $|X|=k$. Since $G$ is saturated, $G+y_{0} x_{0}$ has a $2 k$-cycle $y_{0} x_{1} y_{1} x_{2} y_{2} \ldots x_{k} y_{0}$ where $x_{k}=x_{0}$. Then $G$ contains path $P=y_{0} x_{1} y_{1} x_{2} \ldots x_{k}$.

By the choice of $y_{0},\left\{x_{1}, \ldots, x_{k-1}\right\} \subseteq N\left(y_{0}\right)$. Thus if $x_{k}$ is adjacent to any $y_{j}$ for $1 \leqslant j \leqslant k-2$, then $G$ has cycle $x_{k} y_{j} x_{j} y_{j-1} \ldots y_{0} x_{j+1} y_{j+1} \ldots x_{k}$, a contradiction. Hence $x_{k}$ has only one neighbor on $P$. Let $N_{G}\left(x_{k}\right)=\left\{y_{k-1}, z_{1}, z_{2}, \ldots, z_{s}\right\}$. Since $G$ is 2connected, $s \geqslant 1$. Again, if any $z_{i}$ is adjacent to any $x_{j}$ for $j \leqslant k-2$, then $G$ has cycle $x_{k} z_{i} x_{j} y_{j-1} \ldots y_{0} x_{j+1} y_{j+1} \ldots x_{k}$, a contradiction. Hence $N\left(z_{i}\right)=\left\{x_{k-1}, x_{k}\right\}$ for all $1 \leqslant i \leqslant s$. Switching $z_{1}$ with $y_{k-1}$ we conclude that $N\left(y_{k-1}\right)=\left\{x_{k-1}, x_{k}\right\}$. So, the only vertex of $X-\left\{x_{k}\right\}$ at distance 2 from $x_{k}$ is $x_{k-1}$, a contradiction to Lemma 10 .

Lemma 21. If $G$ is a saturated critical $(X, Y)$-bigraph and some $x_{0} \in X$ has degree 2, then

(a) each of its neighbors is adjacent to all vertices in $X$, and

(b) $d(x) \geqslant 4$ for every $x \in X-\left\{x_{0}\right\}$.

In particular, at most one vertex in $X$ has degree 2.

Proof. Suppose $G$ is a saturated critical $(X, Y)$-bigraph, and $d\left(x_{0}\right)=2$ for some $x_{0} \in X$. Let $N\left(x_{0}\right)=\left\{y_{1}, y_{2}\right\}$. We first prove part (a):

$$
N\left(y_{1}\right)=N\left(y_{2}\right)=X
$$

Indeed, suppose $N\left(y_{j}\right) \neq X$ for some $j \in\{1,2\}$. Then by Lemma 20, $\left|X-N\left(y_{j}\right)\right| \geqslant 2$, say, $\left\{x, x^{\prime}\right\} \subseteq X-N\left(y_{j}\right)$. Consider $A=\left\{x_{0}, x, x^{\prime}\right\}$ and $B=\widehat{N}_{G}(A)$. Then $y_{j} \notin B$ and so $d_{G[A \cup B]}\left(x_{0}\right) \leqslant 1$, a contradiction to (1). This proves (a).

Suppose (b) does not hold and consider an $x \in X-\left\{x_{0}\right\}$ such that $d(x) \leqslant 3$. Note that by $(a), x$ is adjacent to $y_{1}$ and $y_{2}$. For any $x^{\prime} \in X-\left\{x, x_{0}\right\}$, Claim 17 for $A=\left\{x, x^{\prime}, x_{0}\right\}$ yields that there is a common neighbor $y\left(x^{\prime}\right)$ of $x$ and $x^{\prime}$ distinct from $y_{1}$ and $y_{2}$. Since $d(x) \leqslant 3$, all $y\left(x^{\prime}\right)$ coincide, and hence there is a vertex $y$ adjacent to all vertices in $X$ apart from $x_{0}$, a contradiction to Lemma 20. This proves (b).

Lemma 22. If $G$ is a saturated critical $(X, Y)$-bigraph, then for every $y \in Y,|N(y)| \neq$ $|X|-2$. 
Proof. Suppose $G$ is a saturated critical $(X, Y)$-bigraph, and $N\left(y_{0}\right)=X-\left\{x^{\prime}, x^{\prime \prime}\right\}$ for some $y_{0} \in Y$. Let $|X|=k$.

Assume $d\left(x^{\prime}\right) \geqslant d\left(x^{\prime \prime}\right)$. By Lemma $21, d\left(x^{\prime}\right) \geqslant 3$. Since $G$ is saturated, it has a path $P=y_{0} x_{1} y_{1} x_{2} \ldots y_{k-1} x_{k}$ where $x_{k}=x^{\prime}$. We may assume $x^{\prime \prime}=x_{j}$ for some $j$.

If $x_{k}$ is adjacent to any $y_{i}$ for $i \in[k-2]-\{j-1\}$, then $G$ has cycle

$$
y_{0} x_{i+1} y_{i+1} x_{i+2} \ldots x_{k} y_{i} x_{i} \ldots y_{0}
$$

a contradiction. So $N\left(x_{k}\right) \cap V(P) \subseteq\left\{y_{k-1}, y_{j-1}\right\}$. Let $N\left(x_{k}\right)-V(P)=\left\{z_{1}, z_{2}, \ldots, z_{s}\right\}$. Since $d\left(x_{k}\right) \geqslant 3, s \geqslant 1$. Let $T=X-\left\{x_{k}, x_{k-1}, x_{j-1}\right\}$. Again, if any $z_{\ell}$ is adjacent to any $x_{i} \in T$, then $G$ has cycle $y_{0} x_{i+1} y_{i+1} x_{i+2} \ldots x_{k} z_{\ell} x_{i} \ldots y_{0}$, a contradiction. Hence

$$
N\left(z_{\ell}\right) \cap T=\varnothing \quad \text { for all } 1 \leqslant \ell \leqslant s .
$$

Since Claim 19 implies $k \geqslant 6,|T| \geqslant 3$. By Claim 17, for each $x_{i}, x_{i^{\prime}} \in T, G$ contains a 6-cycle $C_{1}$ with $V\left(C_{1}\right) \cap X=\left\{x_{k}, x_{i}, x_{i^{\prime}}\right\}$, say $C_{1}=x_{k} y x_{i} y^{\prime} x_{i^{\prime}} y^{\prime \prime} x_{k}$. By (3) and the fact that $N\left(x_{k}\right) \cap V(P) \subseteq\left\{y_{j-1}, y_{k-1}\right\},\left\{y, y^{\prime \prime}\right\} \subseteq\left\{y_{k-1}, y_{j-1}\right\}$. In particular, $x_{k} y_{j-1} \in E(G)$.

Similarly, if there are $x_{i}, x_{i^{\prime}} \in T$ both not adjacent to $y_{k-1}$ or both not adjacent to $y_{j-1}$, then $G$ does not contain a 6 -cycle $C_{1}$ with $V\left(C_{1}\right) \cap A=\left\{x_{k}, x_{i}, x_{i^{\prime}}\right\}$; however, $G$ is 3-cyclic, a contradiction. This means $\left|N\left(y_{k-1}\right) \cap T\right| \geqslant|T|-1$ and $\left|N\left(y_{j-1}\right) \cap T\right| \geqslant|T|-1$. Since $|T| \geqslant 3$, this implies that there is $x_{i} \in T \cap N\left(y_{k-1}\right) \cap N\left(y_{j-1}\right)$. Note that $i \notin\{j-1, k-1, k\}$, since $x_{j-1} \notin T$. Since $x_{i+1} \notin\left\{x_{j}, x_{k}\right\}, y_{0}$ is adjacent to $x_{i+1}$.
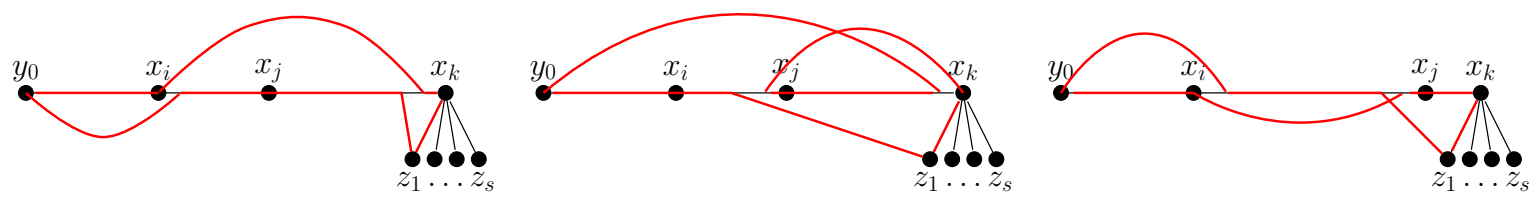

Figure 1: 3 configurations for Lemma 22

Since $G$ is 2-connected, $z_{1}$ has a neighbor in $\left\{x_{k-1}, x_{j-1}\right\}$. If $z_{1} x_{k-1} \in E(G)$, then $G$ has cycle $y_{0} x_{1} \ldots x_{i} y_{k-1} x_{k} z_{1} x_{k-1} y_{k-2} x_{k-2} \ldots x_{i+1} y_{0}$. So $N\left(z_{1}\right)=\left\{x_{k}, x_{j-1}\right\}$ (see Fig. 1 (1)). If $j \neq k-1$, then $G$ has the cycle $y_{0} x_{1} \ldots x_{j-1} z_{1} x_{k} y_{j-1} x_{j} y_{j} x_{j+1} \ldots x_{k-1} y_{0}$ (see Fig. 1 (2)). Hence we may suppose $j=k-1$. Then by the definition of $T, i \leqslant k-3$. So $G$ has the cycle $y_{0} x_{1} \ldots x_{i} y_{k-2} x_{k-1} y_{k-1} x_{k} z_{1} x_{k-2} y_{k-2} \ldots x_{i+1} y_{0}$ (see Fig. 1 (3)), a contradiction.

A critical $(X, Y)$-bigraph $G$ is $Y$-minimal if every proper subgraph $G^{\prime}=\left(X^{\prime}, Y^{\prime} ; E^{\prime}\right)$ of $G$ satisfying (1) is super-cyclic.

Lemma 23. If a saturated critical $Y$-minimal $(X, Y)$-bigraph $G$ has vertices $y_{1}, y_{2} \in Y$ of degree 2 , then $N\left(y_{1}\right) \neq N\left(y_{2}\right)$. 
Proof. Suppose $N\left(y_{1}\right)=N\left(y_{2}\right)=\left\{x_{1}, x_{2}\right\}$, and consider the graph $G^{\prime}:=G-\left\{y_{1}\right\}$ with partite sets $X$ and $Y^{\prime}=Y-\left\{y_{1}\right\}$. Note that in $G$, each cycle of length at least 6 contains at most one vertex in $\left\{y_{1}, y_{2}\right\}$ since the neighbors of such a vertex on the cycle must be exactly $x_{1}$ and $x_{2}$. Hence for each cycle $C$ of length at least 6 in $G$, there exists a cycle $C^{\prime}$ in $G^{\prime}$ with $C \cap X=C^{\prime} \cap X$. We will show that (1) holds for $G^{\prime}$.

Indeed, suppose there exists a set $A \subseteq X$ with $\left|\widehat{N}_{G^{\prime}}(A)\right|<|A|$. Then $\left\{x_{1}, x_{2}\right\} \subseteq A$, $\widehat{N}_{G^{\prime}}(A)=\widehat{N}_{G}(A)-\left\{y_{1}\right\}$, and hence $|\widehat{N}(A)|=|A|$. If $|A| \geqslant 4$, then $\left|\widehat{N}\left(A-\left\{x_{1}\right\}\right)\right| \geqslant$ $\left|A-\left\{x_{1}\right\}\right|=|A|-1$. However, $\widehat{N}\left(A-\left\{x_{1}\right\}\right) \subseteq \widehat{N}(A)-\left\{y_{1}, y_{2}\right\}$, a contradiction. So $|A|=3$, say $A=\left\{x_{1}, x_{2}, x_{3}\right\}, \widehat{N}_{G^{\prime}}(A)=\left\{y_{2}, y_{3}\right\}$, and $\widehat{N}(A)=\left\{y_{1}, y_{2}, y_{3}\right\}$. But there is no 6-cycle in $G$ based on $A$ since $N\left(y_{1}\right)=N\left(y_{2}\right)=\left\{x_{1}, x_{2}\right\}$. This contradicts Claim 17 .

Now suppose $G^{\prime}$ is not 2-connected. Then $G^{\prime}$ contains a cut vertex $v$, and $\left\{v, y_{1}\right\}$ is a cut set in $G$. This implies that $x_{1}$ and $x_{2}$ are in different components of $G-\left\{v, y_{1}\right\}$, and so $v=y_{2}$. Let $x_{3} \in X-\left\{x_{1}, x_{2}\right\}$. Then there is no 6 -cycle based on $\left\{x_{1}, x_{2}, x_{3}\right\}$ in $G$, a contradiction.

By the definition of critical $Y$-minimal bigraphs, $G^{\prime}$ is super-cyclic; but then $G$ also is.

Lemma 24. If $G$ is a saturated critical $Y$-minimal $(X, Y)$-bigraph, $x \in X$ and $C$ is a cycle based on $X-\{x\}$, then $x$ has at least two non-neighbors in $V(C) \cap Y$.

Proof. Let $|X|=k$ and let $C=x_{1} y_{1} \ldots x_{k-1} y_{k-1} x_{1}$. Suppose for the sake of contradiction that $|N(x) \cap V(C)| \geqslant k-2$. If $N(x)$ contains a vertex $y$ that is not in $C$, then because $G$ is 2-connected, $y$ has a neighbor in $V(C)$, say $x_{1}$. Then without loss of generality, $y_{1} \in N(x)$, and we may replace the edge $x_{1} y_{1}$ in $C$ with the path $x_{1} y x y_{1}$ to obtain a cycle based on $X$, a contradiction.

So we may assume $N(x) \subseteq V(C)$. Since $|\widehat{N}(X)| \geqslant|X|$, there exists a vertex $y \in$ $\widehat{N}(X) \backslash V(C)$. Since $G$ is 2-connected and $y x \notin E(G), y$ has some neighbors $x_{i}$ and $x_{j}$ in $C$. If $\left\{y_{i}, y_{j}\right\} \subseteq N(x)$ then we obtain the cycle $x_{1} y_{1} \ldots x_{i} y x_{j} y_{j-1} \ldots y_{i} x y_{j} x_{j+1} \ldots x_{1}$, a contradiction. Similarly, we have that $\left\{y_{i-1}, y_{j-1}\right\} \nsubseteq N(x)$. The remaining case is $N(y)=\left\{x_{i}, x_{i+1}\right\}$ and $N(x)=V(C)-\left\{y_{i}\right\}$. By considering the cycle obtained by replacing $y_{i}$ with $y$, we see that by symmetry, $N\left(y_{i}\right)=\left\{x_{i}, x_{i+1}\right\}$. But this contradicts Lemma 23.

\section{6-cyclic graphs}

In this section, we complete the proof of Theorem 6 by proving that all $(X, Y)$-bigraphs satisfying (1) are 6-cyclic. We will use $N_{C}(x)$ to denote the neighborhoods of $x$ that are in $V(C)$.

Lemma 25. If $G$ is a saturated critical $(X, Y)$-bigraph and $|X|=6$, then $X$ contains a vertex of degree at least 4.

Proof. Suppose all vertices in $X$ have degree at most 3 . 
Case 1: There is a vertex $y \in Y$ with $d(y) \geqslant 4$. By Lemma $22, d(y) \neq 4$, so $d(y) \geqslant 5$. Let $x_{1}, x_{2}, x_{3}, x_{4}, x_{5}$ be five neighbors of $y$; let $C=x_{1} y_{1} x_{2} y_{2} x_{3} y_{3} x_{4} y_{4} x_{5} y_{5} x_{1}$ be a cycle containing them.

If $y \notin V(C)$, then $x_{1}, x_{2}, x_{3}, x_{4}, x_{5}$ each have two neighbors on $C$ and are also adjacent to $y$. Since the degree of each $x_{i}$ is at most 3 , they cannot have any other neighbors. In that case, the set $A=\left\{x_{1}, x_{2}, x_{4}\right\}$ contradicts (1), since $\widehat{N}(A)=\left\{y_{1}, y\right\}$.

Therefore $y \in V(C)$; say, $y=y_{1}$. Then $x_{3}, x_{4}, x_{5}$ have two neighbors on $C$ and an edge to $y_{1}$, so they have degree 3 . By (1) applied to $A=\left\{x_{1}, x_{3}, x_{5}\right\}, x_{1}$ must have an edge to one of $y_{2}, y_{3}, y_{4}$; symmetrically, $x_{2}$ must have an edge to one of $y_{3}, y_{4}, y_{5}$. This yields 3 edges incident to each of $x_{1}, x_{2}, x_{3}, x_{4}, x_{5}$; none of these can have any other neighbors.

By (1), $|\widehat{N}(X)| \geqslant 6$; however, since there is only one vertex in $X-V(C), \widehat{N}(X) \subseteq$ $N(X \cap V(C))=Y \cap V(C)$. This only has size 5, a contradiction.

Case 2: All vertices in $Y$ have degree at most 3. Let $X=\left\{x_{1}, x_{2}, x_{3}, x_{4}, x_{5}, x_{6}\right\}$. Let $C_{1}=x_{1} y_{1} x_{2} y_{2} x_{3} y_{3} x_{1}$ be a 6 -cycle based on $\left\{x_{1}, x_{2}, x_{3}\right\}$ and let $C_{2}=x_{4} y_{4} x_{5} y_{5} x_{6} y_{6} x_{4}$ be a 6 -cycle based on $\left\{x_{4}, x_{5}, x_{6}\right\}$. We have $V\left(C_{1}\right) \cap V\left(C_{2}\right)=\varnothing$, since a vertex in $V\left(C_{1}\right) \cap V\left(C_{2}\right)$ would have degree at least 4 .

In the cycle based on $\left\{x_{1}, x_{2}, x_{4}\right\}$, the vertex $x_{4}$ must have two common neighbors with $\left\{x_{1}, x_{2}\right\}$. Since $\Delta(G) \leqslant 3$, at least one of them is a neighbor of $x_{4}$ on $C_{2}$. Without loss of generality, let $y_{4}$ be a common neighbor with $x_{1}$, so that $x_{1} y_{4} \in E(G)$.

Now consider the cycle based on $\left\{x_{1}, x_{4}, x_{5}\right\}$. By the same argument, either $x_{4}$ or $x_{5}$ must be adjacent to one of $x_{1}$ 's neighbors on $C_{1}$. Without loss of generality, let $x_{4} y_{1}$ be that edge; then the cycle $x_{1} y_{4} x_{5} y_{5} x_{6} y_{6} x_{4} y_{1} x_{2} y_{2} x_{3} y_{3} x_{1}$ is based on $X$, a contradiction.

Claim 26. All $(X, Y)$-bigraphs $G$ satisfying (1) are 6-cyclic.

Proof. Take a vertex-minimal counterexample $G$ with the most edges, meaning in particular that $|X|=6$ and $Y=\widehat{N}(X)$. By Claims $17-19, G$ is $k$-cyclic for $3 \leqslant k \leqslant 5$; therefore $G$ is critical, saturated and $Y$-minimal.

Let $X=\left\{x_{1}, \ldots, x_{6}\right\}$ and $x_{6}$ be a vertex of maximum degree in $X$. By Lemma 25, $d\left(x_{6}\right) \geqslant 4$. Let $C=x_{1} y_{1} x_{2} y_{2} x_{3} y_{3} x_{4} y_{4} x_{5} y_{5} x_{1}$ be a cycle based on $X-\left\{x_{6}\right\}$. By Lemma 16 and Lemma 24, $x_{6}$ has either 2 or 3 neighbors on $C$, so it has at least one neighbor $y_{6}$ not on $C$.

By symmetry, the following two cases are exhaustive.

Case 1: $\left\{y_{1}, y_{3}\right\} \subseteq N_{C}\left(x_{6}\right)$. In this case, by Claim 14, no vertex $y \in N\left(x_{6}\right)-V(C)$ can be adjacent to $x_{1}, x_{2}, x_{3}$, or $x_{4}$, so it must be adjacent to $x_{5}$ and $x_{6}$ only. By Lemma 23, $y_{6}$ is the only such vertex. By Claim 14 again, $x_{6}$ cannot be adjacent to $y_{4}$ or $y_{5}$. To have $d\left(x_{6}\right) \geqslant 4, x_{6}$ must also be adjacent to $y_{2}$, and therefore $d\left(x_{6}\right)=4$.

If $x_{2} y_{4} \in E(G)$, then the cycle $x_{2} y_{4} x_{4} \ldots y_{2} x_{6} y_{6} x_{5} y_{5} x_{1} y_{1} x_{2}$ is based on $X$, and if $x_{2} y_{5} \in E(G)$, the cycle $x_{2} y_{2} \ldots x_{5} y_{6} x_{6} y_{1} x_{1} y_{5} x_{2}$ is based on $X$. Thus, $x_{2} y_{4} \notin E(G)$ and $x_{2} y_{5} \notin E(G)$. A similar argument shows that $x_{3} y_{5}, x_{3} y_{4} \notin E(G)$. However, applying Claim 17 to $A=\left\{x_{2}, x_{3}, x_{5}\right\}$, we find distinct vertices $y^{\prime} \in N\left(x_{2}\right) \cap N\left(x_{5}\right)$ and $y^{\prime \prime} \in$ $N\left(x_{3}\right) \cap N\left(x_{5}\right)$ such that $y^{\prime}, y^{\prime \prime} \notin\left\{y_{4}, y_{5}, y_{6}\right\}$. Therefore $x_{5}$ is adjacent to $y_{4}, y_{5}, y_{6}, y^{\prime}, y^{\prime \prime}$, and $d\left(x_{5}\right) \geqslant 5>d\left(x_{6}\right)$, contradicting the choice of $x_{6}$. 
Case 2: $N_{C}\left(x_{6}\right)=\left\{y_{1}, y_{2}\right\}$. In this case, in order to have $d\left(x_{6}\right) \geqslant 4, x_{6}$ must have neighbors $y, y^{\prime}$ outside $C$. By Claim 14, $y$ and $y^{\prime}$ can only have $x_{4}$ and $x_{5}$ as neighbors. If $y$ is adjacent to $x_{4}$ and $y^{\prime}$ is adjacent to $x_{5}$, or vice versa, we contradict Claim 15; if both are adjacent only to $x_{4}$ or both only to $x_{5}$, we contradict Lemma 23 .

\section{Bigraphs with high minimum degree}

\subsection{Properties of smallest counterexamples}

Throughout this subsection, we assume that $G$ is a vertex-minimal counterexample to Theorem 8 with the most edges; let $G \in \mathcal{G}(n, m, \delta)$ where $\delta \geqslant \max \left\{n, \frac{m+10}{4}\right\}$. Then for each $X^{\prime} \subset X$ with $X^{\prime} \neq X, G\left[X^{\prime} \cup Y\right]$ also satisfies the conditions of Theorem 8 and hence is super-cyclic.

Let $G^{\prime}=G[X \cup \widehat{N}(X)]$, i.e., $G^{\prime}$ is obtained by removing only the degree-1 vertices of $G$. Then $G^{\prime}$ is critical and saturated. In particular, for every $x \in X$, there exists a cycle $C$ in $G^{\prime}$ (and therefore in $G$ ) such that $V(C) \cap X=X-\{x\}$. By Lemma 12, $G^{\prime}$ has an $x, V(C)$-fan $F$ of size 2 .

Among the triples $(C, x, F)$ where $x \in X, C$ is a cycle with $V(C) \cap X=X-\{x\}$ and $F$ is an $x, V(C)$-fan, choose a triple such that the size of $F$ is maximized, and subject to this, $|V(F)|$ is minimized. Let $|V(C)|=2 \ell$ (so $|X|=\ell+1$ ). Let $t$ be the size of $F$, and let $T=V(C) \cap V(F)=\left\{u_{1}, \ldots, u_{t}\right\}$.

Fix a clockwise direction of $C$. For every vertex $u$ of $C, x_{C}^{+}(u)$ (respectively, $x_{C}^{-}(u)$ ) denotes the closest to $u$ clockwise (respectively, counterclockwise) vertex of $X$ distinct from $u$. For a set $U \subset V(C), X_{C}^{+}(U)=\left\{x_{C}^{+}(u): u \in U\right\}$. When $C$ is clear from the content, the subscripts could be omitted. The vertices $y^{+}(u), y^{-}(u)$ and the sets $X^{-}(U), Y^{+}(U), Y^{-}(U)$ are defined similarly.

Viewing $F$ as a tree (spider) with root $x$, any two vertices $u, v \in V(F)$ define the unique $u, v$-path $F[u, v]$ in $F$. For $u, v \in V(C)$, let $C[u, v]$ be the clockwise $u, v$-path in $C$ and let $C^{-}[u, v]$ be the counterclockwise $u, v$-path in $C$.

Lemma 27. $t \leqslant \ell-2$.

Proof. We first show that

$$
t \leqslant \ell-|T \cap X|
$$

If $w \in T \cap X$ and $y^{+}(w) \in T$, then the cycle $w F\left[w, y^{+}(w)\right] y^{+}(w) C\left[y^{+}(w), w\right] w$ is based on $X$, a contradiction. Similarly, $y^{-}(w), x^{+}(w), x^{-}(w) \notin T$. Thus, $|T \cap X| \leqslant \ell / 2$ and $|T \cap Y| \leqslant \ell-2|T \cap X|$. This proves (4).

For the remainder of the proof, note that if Claims $13-15$ are applied to $G^{\prime}$, then the conclusions hold for $G$ as well, since they are unaffected by the addition of vertices of degree 1 in $Y$.

Let $C=x_{1} y_{1} \ldots x_{\ell} y_{\ell} x_{1}$, and suppose $t \geqslant \ell-1$. By (4), $|T \cap X| \leqslant 1$. If $T \cap X=\varnothing$, we may assume that $x y_{i} \in E(G)$ for all $1 \leqslant i \leqslant \ell-1$. By $(1),|\widehat{N}(X)| \geqslant \ell+1$, so there is $y \in Y-V(C)$ with at least two neighbors in $X$. This will contradict one of Claims 13-15 (possibly, in reversed orientation of $C$ ), unless all such $y$ are adjacent to 
only $x_{\ell}=x^{-}\left(y_{\ell}\right)$ and $x_{1}=x^{+}\left(y_{\ell}\right)$. Fix such a vertex $y$. Let $A=X-\left\{x_{\ell}\right\}$. There exists a vertex $y^{\prime} \in(Y-V(C)) \cup\left\{y_{\ell}\right\}$ such that $y^{\prime} \in \widehat{N}(A)$, i.e., $y^{\prime}$ has two neighbors other than $x_{\ell}$ (so $\left.y^{\prime} \neq y\right)$. Let $C^{\prime}$ be the cycle obtained by replacing $y_{\ell}$ with $y$. Then the vertex $y^{\prime}$ violates one of Claims $13-15$ with respect to $C^{\prime}$.

If $|T \cap X|=1$, then by (4), we may assume that $x y_{i} \in E(G)$ for all $1 \leqslant i \leqslant \ell-2$ and that $x$ has a common neighbor $y \in Y-V(C)$ with $x_{\ell}$. By $(1),\left|\widehat{N}\left(X-x_{\ell}\right)\right| \geqslant \ell$, so there is $y_{0} \in(Y-V(C)) \cup\left\{y_{\ell-1}, y_{\ell}\right\}$ with at least two neighbors in $X-x_{\ell}$. If $y_{0} \in(Y-V(C))$, this again will contradict one of Claims 13-15, unless $N\left(y_{0}\right)=\left\{x_{\ell-1}, x_{1}\right\}$. In this case, we obtain the longer cycle $y_{1} C\left[y_{1}, y_{\ell-2}\right] y_{\ell-2} x y x_{\ell} y_{\ell-1} x_{\ell-1} y_{0} x_{1}$. So suppose without loss of generality $y_{0}=y_{\ell}$ has a neighbor $z \in X-\left\{x_{\ell}, x_{1}\right\}$. By the case, $z \neq x$, so suppose $z=x_{j}$ for some $2 \leqslant j \leqslant \ell-1$. Then $G$ has cycle $y_{\ell} C\left[y_{\ell}, y_{j-1}\right] y_{j-1} x y x_{\ell} C^{-}\left[x_{\ell}, x_{j}\right] x_{j} y_{\ell}$ based on $X$, a contradiction.

Given a cycle $C$ and distinct $x_{1}, x_{2}, x_{3} \in X \cap V(C)$, we say that $x_{1}$ and $x_{2}$ cross at $x_{3}$ if the cyclic order is $x_{1}, x_{3}, x_{2}$ and $x_{1} y^{+}\left(x_{3}\right), x_{2} y^{-}\left(x_{3}\right) \in E(G)$ or if the cyclic order is $x_{1}, x_{2}, x_{3}$ and $x_{1} y^{-}\left(x_{3}\right), x_{2} y^{+}\left(x_{3}\right) \in E(G)$. In this case, we also say that $x_{3}$ is crossed $b y$ $x_{1}$ and $x_{2}$.

The following is Lemma 2.8 in [9]. It holds for each bipartite graph $G$ (no restrictions).

Lemma $28([9])$. Let $C$ be a cycle of an $(X, Y)$-bigraph $G$, and let $u, v \in V(C) \cap X$. If $u$ and $v$ have at most $r$ crossings, then $d_{C}(u)+d_{C}(v) \leqslant|V(C)| / 2+2+r$.

Proof. We induct on $r$. Suppose $r=0$. Consider the two paths $P_{1}=C[u, v]$ and $P_{2}=C^{-}[u, v]$. In $P_{1}=v_{1} \ldots v_{k}\left(v_{1}=u, v_{k}=v\right)$, each $v_{i} \in X$ satisfies at most one of the following: $v_{i+1} u \in E(G)$ or $v_{i-1} v \in E(G)$. So $d_{P_{1}}(u)+d_{P_{1}}(v) \leqslant\left|V\left(P_{1}\right) \cap X\right|$. Similarly, $d_{P_{2}}(u)+d_{P_{2}}(v) \leqslant\left|V\left(P_{2}\right) \cap X\right|$. Since $\left(X \cap V\left(P_{1}\right)\right) \cap\left(X \cap V\left(P_{2}\right)\right)=\{u, v\}$ and $V\left(P_{1}\right) \cup V\left(P_{2}\right)=V(C)$, we get $d_{C}(u)+d_{C}(v) \leqslant|V(C)| / 2+2$.

For $r \geqslant 1$, delete an edge incident to $u$ that is used in a crossing, and induct.

Lemma 29. If $u_{i} \in X \cap T$, then $y^{+}\left(u_{i}\right)$ has no neighbors in $(F-V(C)) \cup X^{+}(T) \backslash\left\{x^{+}\left(u_{i}\right)\right\}$.

Proof. Suppose $y^{+}\left(u_{i}\right)$ has a neighbor $z$ in $F-V(C)$. Then the cycle

$$
u_{i} F\left[u_{i}, z\right] z y^{+}\left(u_{i}\right) C\left[y^{+}\left(u_{i}\right), u_{i}\right] u_{i}
$$

is based on $X$, a contradiction.

Suppose now that $y^{+}\left(u_{i}\right)$ has a neighbor $x_{1}$ in $X^{+}(T) \backslash\left\{x^{+}\left(u_{i}\right)\right\}$, where $u \in T$ satisfies $x^{+}(u)=x_{1}$. Then the cycle $x_{1} y^{+}\left(u_{i}\right) C\left[y^{+}\left(u_{i}\right), u\right] u F\left[u, u_{i}\right] u_{i} C^{-}\left[u_{i}, x_{1}\right] x_{1}$ is based on $X$, a contradiction.

Lemma 30. If $x_{1} \in X^{+}(T)$, then $x_{1}$ cannot have a neighbor in $F-V(C)$.

Proof. Suppose $x_{1}$ has a neighbor $y^{\prime}$ in $F-V(C)$. Let $u_{1} \in T$ be such that $x_{1}=x^{+}\left(u_{1}\right)$ and $z$ be a neighbor of $u_{1}$ in $F$. Let $P$ be a $z, y^{\prime}$-path in $F$ and the cycle $C^{\prime}$ be defined by $C^{\prime}=x_{1} C\left[x_{1}, u_{1}\right] u_{1} z P y^{\prime} x_{1}$. If $y^{\prime} \neq z$, then $C^{\prime}$ is based on $X$ and we are done. Thus $z=y^{\prime}$ and hence $u_{1} \in X$. Let $F^{\prime}=F-u_{1}$. Note that $F^{\prime}$ is an $x, V\left(C^{\prime}\right)$-fan such that $|V(F \cap C)|=\left|V\left(F^{\prime} \cap C^{\prime}\right)\right|$, but $\left|V\left(F^{\prime}\right)\right|<|V(F)|$, contradicting the choice of $C$ and $F$. 
Lemma 31. Suppose that $x_{1}, x_{2} \in X^{+}(T)$. Then

(i) $x_{1}$ and $x_{2}$ share no neighbors in $Y-V(C)$;

(ii) neither $x_{1}$ nor $x_{2}$ share a neighbor in $Y-V(C)$ with $x$.

Proof. Part (i) follows from Claim 13. From Lemma 30, if $x_{1}$ and $x$ have a common neighbor outside of $C$, it is not in $F$. Suppose they share some neighbor $y \in Y-V(C)$. Let $x_{1}=x^{+}\left(u_{1}\right)$. Then we have a longer cycle $x_{1} C\left[x_{1}, u_{1}\right] u_{1} F\left[u_{1}, x\right] x y x_{1}$. The same holds for $x_{2}$ and $x$. This proves (ii).

Lemma 32. Suppose $u_{1}, u_{2} \in T$. If $x_{1}=x^{+}\left(u_{1}\right)$ and $x_{2}=x^{+}\left(u_{2}\right)$ cross at $x_{3} \in X \cap V(C)$, then

(i) $x_{3} \notin T$;

(ii) $G$ has a cycle $C^{\prime}$ containing $\left(X \cap V(C)-\left\{x_{3}\right\}\right) \cup\{x\}$ such that $\left|C^{\prime}\right| \geqslant|C|$;

(iii) $x_{3}$ shares no neighbors in $Y-V(C)$ with any vertex in the set $\{x\} \cup X^{+}(T)$;

(iv) $x_{3}$ has at most $t$ neighbors on $C$.

Proof. Suppose that the cyclic order is $x_{1}, x_{3}, x_{2}$ and $x_{1} y^{+}\left(x_{3}\right), x_{2} y^{-}\left(x_{3}\right) \in E(G)$ (the other case is symmetric).

For part (i), let $y$ be a neighbor of $x_{3}$ in $F$. Let $z$ be a neighbor of $u_{1}$ in $F$. Let $P$ be a $z, y$-path in $F$ and the cycle $C^{\prime}$ be defined by

$$
C^{\prime}:=x_{1} y^{+}\left(x_{3}\right) C\left[y^{+}\left(x_{3}\right), u_{1}\right] u_{1} z P y x_{3} C^{-}\left[x_{3}, x_{1}\right] x_{1} \text {. }
$$

Then $C^{\prime}$ is based on $X$. This contradiction proves (i).

The cycle

$$
C_{1}:=x_{1} y^{+}\left(x_{3}\right) C\left[y^{+}\left(x_{3}\right), u_{2}\right] u_{2} F\left[u_{2}, u_{1}\right] u_{1} C^{-}\left[u_{1}, x_{2}\right] x_{2} y^{-}\left(x_{3}\right) C^{-}\left[y^{-}\left(x_{3}\right), x_{1}\right] x_{1}
$$

proves (ii).

To prove (iii), assume that $y \in Y-V(C)$ is a common neighbor of $x_{3}$ and a vertex in $\{x\} \cup X^{+}(T)$, and consider all cases. If $y x \in E(G)$, let

$$
C^{\prime}=x_{1} y^{+}\left(x_{3}\right) C\left[y^{+}\left(x_{3}\right), u_{1}\right] u_{1} F\left[u_{1}, x\right] x y x_{3} C^{-}\left[x_{3}, x_{1}\right] x_{1} .
$$

If $y$ is not in $F\left[x, u_{1}\right]$, then $C^{\prime}$ is a cycle based on $X$, a contradiction. Otherwise, let $F^{\prime \prime}$ be $F-F\left[u_{1}, y\right]$. Note $F^{\prime \prime}$ is an $x, V\left(C^{\prime \prime}\right)$-fan where

$$
C^{\prime \prime}=x_{1} y^{+}\left(x_{3}\right) C\left[y^{+}\left(x_{3}\right), u_{1}\right] u_{1} F\left[u_{1}, y\right] y x_{3} C^{-}\left[x_{3}, x_{1}\right] x_{1},
$$

and $|V(F \cap C)|=\left|V\left(F^{\prime \prime} \cap C^{\prime \prime}\right)\right|$, but $\left|V\left(F^{\prime \prime}\right)\right|<|V(F)|$, contradicting the choice of $C$ and $F$. 
If $u_{j} \in T, x_{j}=x^{+}\left(u_{j}\right), y x_{j} \in E(G)$, and $x_{j} \in C\left[y^{+}\left(x_{3}\right), u_{1}\right]$, then the cycle

$$
C^{\prime}:=x_{1} C\left[x_{1}, x_{3}\right] x_{3} y x_{j} C\left[x_{j}, u_{1}\right] u_{1} F\left[u_{1}, u_{j}\right] u_{j} C^{-}\left[u_{j}, y^{+}\left(x_{3}\right)\right] y^{+}\left(x_{3}\right) x_{1}
$$

is based on $X$. Similarly, if $x_{j} \in C\left[u_{1}, y^{-}\left(x_{3}\right)\right]$, then the cycle

$$
C^{\prime}:=x_{2} C\left[x_{2}, u_{j}\right] u_{j} F\left[u_{j}, u_{2}\right] u_{2} C^{-}\left[u_{2}, x_{3}\right] x_{3} y x_{j} C\left[x_{j}, y^{-}\left(x_{3}\right)\right] y^{-}\left(x_{3}\right) x_{2}
$$

is based on $X$, a contradiction. This proves (iii).

By the choice of $(C, x, F)$ and (ii), $x_{3}$ has at most $t$ neighbors on $C_{1}$. The only vertices in $Y \cap V(C)-V\left(C_{1}\right)$ are $y^{-}\left(x_{1}\right)$ and $y^{-}\left(x_{2}\right)$. If $x_{3} y^{-}\left(x_{1}\right) \in E(G)$, then the cycle

$$
y^{-}\left(x_{1}\right) C\left[y^{-}\left(x_{1}\right), y^{-}\left(x_{3}\right)\right] y^{-}\left(x_{3}\right) x_{2} C\left[x_{2}, u_{1}\right] u_{1} F\left[u_{1}, u_{2}\right] u_{2} C^{-}\left[u_{2}, x_{3}\right] x_{3} y^{-}\left(x_{1}\right)
$$

is based on $X$. If $x_{3} y^{-}\left(x_{2}\right) \in E(G)$, then the cycle

$$
x_{1} C\left[x_{1}, x_{3}\right] x_{3} y^{-}\left(x_{2}\right) C\left[y^{-}\left(x_{2}\right), u_{1}\right] u_{1} F\left[u_{1}, u_{2}\right] u_{2} C^{-}\left[u_{2}, y^{+}\left(x_{3}\right)\right] y^{+}\left(x_{3}\right) x_{1}
$$

is based on $X$. This proves (iv).

Lemma 33. Suppose $u_{1}, u_{2} \in T, x_{1}=x^{+}\left(u_{1}\right)$, and $x_{2}=x^{+}\left(u_{2}\right)$. Then no two vertices $x_{3}, x_{4} \in V(C)$ crossed by $x_{1}$ and $x_{2}$ have a shared neighbor in $Y-V(C)$.

Proof. Suppose vertices $x_{3}, x_{4} \in V(C) \cap X$ are crossed by $x_{1}$ and $x_{2}$ and there is some $y \in\left(N\left(x_{3}\right) \cap N\left(x_{4}\right)\right)-V(C)$. By Lemma 32, $y \notin V(F)$.

We consider two cases. If $x_{3}$ and $x_{4}$ both are on $C\left[x_{1}, x_{2}\right]$ or both are on $C\left[x_{2}, x_{1}\right]$, then we may assume that their cyclic order is $x_{1}, x_{3}, x_{4}, x_{2}$. In this case, the cycle

$$
x_{1} C\left[x_{1}, x_{3}\right] x_{3} y x_{4} C\left[x_{4}, u_{2}\right] u_{2} F\left[u_{2}, u_{1}\right] u_{1} C^{-}\left[u_{1}, x_{2}\right] x_{2} y^{-}\left(x_{4}\right) C^{-}\left[y^{-}\left(x_{4}\right), y^{+}\left(x_{3}\right)\right] y^{+}\left(x_{3}\right) x_{1}
$$

is based on $X$.

If one of $x_{3}$ and $x_{4}$ is on $C\left[x_{1}, x_{2}\right]$ and the other is on $C\left[x_{2}, x_{1}\right]$, then we may assume that their cyclic order is $x_{1}, x_{3}, x_{2}, x_{4}$. In this case, the cycle

$$
x_{1} C\left[x_{1}, x_{3}\right] x_{3} y x_{4} C^{-}\left[x_{4}, x_{2}\right] x_{2} y^{+}\left(x_{4}\right) C\left[y^{+}\left(x_{4}\right), u_{1}\right] u_{1} F\left[u_{1}, u_{2}\right] u_{2} C^{-}\left[u_{2}, y^{+}\left(x_{3}\right)\right] y^{+}\left(x_{3}\right) x_{1}
$$

is based on $X$. This proves the lemma.

Lemma 34. Let $A \subseteq X^{+}(T)$. Then $\sum_{w \in A} d_{C}(w) \leqslant|A|(\ell-2)+2$.

Proof. Let $x_{1}, x_{2} \in A$ such that $x_{1}=x^{+}\left(u_{1}\right)$ and $x_{2}=x^{+}\left(u_{2}\right)$ for some $u_{1}, u_{2} \in T$. We first prove that

$$
\text { if } u_{2} \in Y \text { and } y^{+}\left(x_{2}\right) x_{1} \in E(G) \text {, then } d_{C}\left(x_{2}\right) \leqslant \ell-2 \text {. }
$$

The cycle $C^{\prime}=x_{1} y^{+}\left(x_{2}\right) C\left[y^{+}\left(x_{2}\right), u_{1}\right] u_{1} F\left[u_{1}, u_{2}\right] u_{2} C^{-}\left[u_{2}, x_{1}\right] x_{1}$ contains all vertices in $C$ except $x_{2}$ and possibly $y^{+}\left(u_{1}\right)$ (in the case that $u_{1} \in X$ ). By Lemma 29 and Lemma 30, $N_{C}\left(x_{2}\right)=N_{C^{\prime}}\left(x_{2}\right)$. By Lemma 27 applied to $C^{\prime}$ and $x_{2}, d_{C}\left(x_{2}\right)=d_{C^{\prime}}\left(x_{2}\right) \leqslant \ell-2$. 
In particular, if $d_{C}\left(x_{1}\right)=\ell$, i.e., $x_{1}$ is adjacent to every $y$ vertex in $C$, then by Lemma 29, each $x_{2} \in X^{+}(T)-\left\{x_{1}\right\}$ satisfies $u_{2} \in Y$. Therefore by $(5), d_{C}\left(x_{2}\right) \leqslant \ell-2$. It follows that $\sum_{w \in A} d_{C}(w) \leqslant|A|(\ell-2)+2$.

So suppose every $w \in A$ has $d_{C}(w) \leqslant \ell-1$, and there exist two vertices $x_{1}, x_{2} \in A$ with equality. Define $u_{1}, u_{2}$ as before. Then for every $x_{3} \in X^{+}(T)-\left\{x_{1}, x_{2}\right\}$, either $u_{1} \in Y$ and $x_{3} y^{+}\left(x_{1}\right) \notin E(G)$ by $(5)$, or $u_{1} \in X$ and $x_{3} y^{+}\left(x_{1}\right) \notin E(G)$ by Lemma 29 . The same holds for $x_{3}$ and $x_{2}$. Therefore $d_{C}\left(x_{3}\right) \leqslant \ell-2$, and again $\sum_{w \in A} d_{C}(w) \leqslant|A|(\ell-2)+2$.

Lemma 35. Suppose $t \geqslant 4, u_{1}, u_{2} \in T, x_{1}=x^{+}\left(u_{1}\right)$, and $x_{2}=x^{+}\left(u_{2}\right)$. Then at most one vertex in $C$ is crossed by $x_{1}$ and $x_{2}$.

Proof. Suppose vertices $x_{3}, x_{4} \in V(C) \cap X$ are crossed by $x_{1}$ and $x_{2}$.

Let $A=X^{+}(T) \cup\left\{x, x_{3}, x_{4}\right\}$ (possibly, $X^{+}(T) \cap\left\{x_{3}, x_{4}\right\} \neq \varnothing$ ), and $A^{\prime}=A-\left\{x, x_{3}, x_{4}\right\}$. Note that $\left|A^{\prime}\right| \geqslant t-2$, and by Lemma 34 applied to $A^{\prime}, \sum_{w \in A^{\prime}} d_{C}(w) \leqslant\left|A^{\prime}\right|(\ell-2)+2$.

Since $x$ can have at most $t$ neighbors on $C,|N(x)-V(C)| \geqslant \delta-t$. By Lemma 32(iv), $\left|N\left(x_{3}\right)-V(C)\right| \geqslant \delta-t$ and $\left|N\left(x_{4}\right)-V(C)\right| \geqslant \delta-t$. By Claims 13-15 (applied to $G^{\prime}$ ) and Lemmas 32(iii) and 33, no two distinct vertices in $A$ have a common neighbor in $Y-V(C)$. Thus, remembering the $\ell$ vertices in $Y \cap V(C)$, we get

$$
\begin{aligned}
|Y| & \geqslant \ell+\sum_{u \in A}|N(u)-V(C)| \\
& =\ell+|N(x)-V(C)|+\left|N\left(x_{3}\right)-V(C)\right|+\left|N\left(x_{4}\right)-V(C)\right|+\sum_{u \in A^{\prime}}|N(u)-V(C)| \\
& \geqslant \ell+3(\delta-t)+\delta\left|A^{\prime}\right|-(\ell-2)\left|A^{\prime}\right|-2 \\
& \geqslant \ell+3 \delta-3 t+(\delta-\ell+2)\left|A^{\prime}\right|-2 \\
& \geqslant \ell+3 \delta-3 t+(\delta-\ell+2)(t-2)-2 \\
& \geqslant \ell+3 \delta-3 t+(\delta-\ell+2)+(\delta-\ell+2)(t-3)-2 \\
& \geqslant \ell+3 \delta-3 t+(\delta-\ell+2)+3(t-3)-2 \\
& =4 \delta-3 t+2+3(t-3)-2=4 \delta-9,
\end{aligned}
$$

as claimed.

Lemma 36. For any $x_{1}, x_{2} \in X, x_{1}$ and $x_{2}$ cannot be separated by a set of two vertices.

Proof. Recall that $G$ is a vertex-minimum counterexample to Theorem 8, and $G^{\prime}=G[X \cup$ $\widehat{N}(X)]$ is critical and saturated.

Suppose that for some $x_{1}, x_{2} \in X, u_{1}, u_{2} \in V(G), x_{1}$ and $x_{2}$ are in different components of $G-\left\{u_{1}, u_{2}\right\}$. Note that $u_{1}, u_{2} \in V\left(G^{\prime}\right)$, since $V(G)-V\left(G^{\prime}\right)$ contains only vertices of degree 1 in $Y$.

If there also exists $x_{3} \in X-\left\{x_{1}, x_{2}\right\}$ such that $x_{3}$ is in a different component of $G-\left\{u_{1}, u_{2}\right\}$ than both $x_{1}$ and $x_{2}$, then $G$ cannot contain a 6 -cycle based on $\left\{x_{1}, x_{2}, x_{3}\right\}$, since these vertices are separated by a set of size two. Hence we may assume $G-\left\{u_{1}, u_{2}\right\}$ contains exactly two components containing vertices in $X$. Call these components $D_{1}$ and $D_{2}$ where $x_{1} \in V\left(D_{1}\right)$ and $x_{2} \in V\left(D_{2}\right)$. 
Choose any two vertices $x, x^{\prime} \in X-\left\{u_{1}, u_{2}\right\}$; then choose a third vertex $x^{\prime \prime} \in X-$ $\left\{u_{1}, u_{2}\right\}$ such that not all three of $x, x^{\prime}, x^{\prime \prime}$ are in the same component of $G-\left\{u_{1}, u_{2}\right\}$. Let $C$ be a cycle based on $A=\left\{x, x^{\prime}, x^{\prime \prime}\right\}$.

Since $\left\{u_{1}, u_{2}\right\}$ separates one of the vertices of $A$ from the others, $u_{1}, u_{2} \in V(C)$; since $V(C) \cap X=A$ and neither $u_{1}$ nor $u_{2}$ is in $A$, we must have $u_{1}, u_{2} \in Y$.

Moreover, $u_{1}$ must have an edge to either $x$ or $x^{\prime}$ in $C$, and therefore in $G$. Since $x, x^{\prime} \in X$ were arbitrary, $\left|N\left(u_{1}\right)\right| \geqslant|X|-1$. By Lemma 20 applied to $G^{\prime}, N_{G^{\prime}}\left(u_{1}\right)=X$, and therefore $N\left(u_{1}\right)=X$. By symmetry, we also obtain $N\left(u_{2}\right)=X$.

Now suppose each component of $G-\left\{u_{1}, u_{2}\right\}$ has at least 2 vertices in $X$. For $i \in$ $\{1,2\}$, set $X_{i}=X \cap D_{i}$. By the minimality of $G$, there exists a cycle $C_{1}$ of $G$ based on $X_{1} \cup\left\{x_{2}\right\}$ and a cycle $C_{2}$ based on $X_{2} \cup\left\{x_{1}\right\}$. Since $D_{1}$ and $D_{2}$ are separated by $\left\{u_{1}, u_{2}\right\}$, $N_{C_{1}}\left(x_{2}\right)=N_{C_{2}}\left(x_{1}\right)=\left\{u_{1}, u_{2}\right\}$. Therefore $\left(C_{1}-\left\{x_{2}\right\}\right) \cup\left(C_{2}-\left\{x_{1}\right\}\right)$ is a cycle in $G$ which is based on $X$, a contradiction.

Thus we may assume without loss of generality that $V\left(D_{1}\right) \cap X=\left\{x_{1}\right\}$. Note that this implies all neighbors of $x_{1}$ other than $u_{1}$ and $u_{2}$ have degree 1 . Let $G_{1}$ be obtained from $G$ by deleting $x_{1}$ and all of its neighbors except for $u_{1}$.

We will show that $G_{1}$ is a counterexample that has fewer vertices than $G$. Set $X^{\prime}=$ $X-\left\{x_{1}\right\}=X \cap V\left(G_{1}\right)$. If there exists $A \subseteq X^{\prime}$ with $|A| \geqslant 3$ such that $\left|\widehat{N}_{G_{1}}(A)\right|<|A|$, then in $G, \widehat{N}_{G}\left(A \cup\left\{x_{1}\right\}\right)=\widehat{N}_{G_{1}}(A) \cup\left\{u_{2}\right\}<\left|A \cup\left\{x_{1}\right\}\right|$, a contradiction.

Next, we will show that for all $A$ with $|A| \geqslant 3, G_{1}\left[A \cup \widehat{N}_{G_{1}}(A)\right]$ is 2-connected. Recall that $G_{1}-\left\{u_{1}\right\}=D_{2}$. The subgraph of $D_{2}$ obtained by removing all vertices in $Y$ of degree 1 is still connected. Call this subgraph $H$. If $A=X^{\prime}$, then $G_{1}\left[A \cup \widehat{N}_{G_{1}}(A)\right]=G_{1}\left[H \cup\left\{u_{1}\right\}\right]$. Since $H$ is connected and $u_{1}$ is adjacent to all vertices in $X^{\prime}, G_{1}\left[H \cup\left\{u_{1}\right\}\right]$ is 2-connected. Now suppose $A \neq X^{\prime}$. Then by the choice of $G$ as a minimum counterexample, there exists a cycle $C$ in $G$ with $V(C) \cap X=A \cup\left\{x_{1}\right\}$, where $N_{C}\left(x_{1}\right)=\left\{u_{1}, u_{2}\right\}$. In particular, $P:=C-\left\{x_{1}, u_{1}, u_{2}\right\}$ is a path containing all vertices of $A$. In $G_{1}, G_{1}\left[A \cup \widehat{N}_{G_{1}}(A)\right]$ can be obtained from $P$ by adding $u_{1}$, which is adjacent to all of $V(P) \cap X$, and possibly adding some additional vertices in $Y$ with degree at least 2 . Hence it is 2-connected.

Next, suppose that it is super-cyclic. By the minimality of $G, G$ contains no cycle $C$ based on $X$; however, because $G_{1}$ is super-cyclic, we may find a cycle $C^{\prime}=v_{1} v_{2} \ldots v_{2\left|X^{\prime}\right|} v_{1}$ in $G_{1}$ (and therefore in $G$ ) based on $X-\left\{x_{1}\right\}$ such that $u_{2} \notin V\left(C^{\prime}\right)$. If $u_{1} \notin V\left(C^{\prime}\right)$, then we may replace in $C^{\prime}$ any segment $v_{i} v_{i+1} v_{i+2}$ (for $v_{i} \in X$ ) with the path $v_{i} u_{1} x_{1} u_{2} v_{i+2}$ to obtain a contradiction. Otherwise, if $u_{1}=v_{i}$ for some $i$, we replace the path $v_{i-1} u_{1} v_{i+2}$ with $v_{i-1} u_{1} x u_{2} v_{i+2}$.

Finally, we have $\left|Y \cap G_{1}\right| \leqslant|Y|-(\delta-1) \leqslant(4 \delta-10)-(\delta-1) \leqslant 4(\delta-1)-10$. The last inequality holds because we may assume that $|X| \geqslant 7$ and therefore $\delta \geqslant 7$, since Theorem 6 implies Theorem 8 for $|X| \leqslant 6$. This shows that $G_{1}$ is a counterexample for Theorem 8 (with $\delta^{\prime}=\delta-1$ ) which has fewer vertices than $G$, contradicting the choice of G. 


\subsection{Proof of Theorem 8}

Proof of Theorem 8. As in the previous subsection, suppose for the sake of contradiction that $G$ is a vertex-minimum, edge-maximal counterexample to Theorem 8. By the choice of $G$, for each $x \in X$, there exists some cycle $C$ with $V(C) \cap X=X-\{x\}$. We may also assume that $|X| \geqslant 7$ and therefore $\delta \geqslant 7$, since Theorem 6 implies Theorem 8 for $|X| \leqslant 6$.

Letting $G^{\prime}=G[X \cup \widehat{N}(X)]$, it follows from our choice of $G$ that $G^{\prime}$ is critical and saturated.

If there exists a pair $(x, C)$ with an $x, V(C)$-fan $F$ of size at least 4 , then choose such a triple which maximizes $t=|V(F) \cap V(C)|$, and subject to this, minimizes $|V(F)|$. Let $T=V(F) \cap V(C)$. By Lemmas 31 and 35, no two vertices in $X^{+}(T) \cup\{x\}$ share a neighbor outside of $V(C)$, and no two vertices in $X^{+}(T)$ cross more than one time. By Lemma 28, for each pair $x_{1}, x_{2} \in X^{+}(T), d_{C}\left(x_{1}\right)+d_{C}\left(x_{2}\right) \leqslant|V(C) \cap Y|+3=|X|+2$. Therefore

$$
\begin{aligned}
|Y| & \geqslant|V(C) \cap Y|+\sum_{w \in X^{+}(T) \cup\{x\}} d_{Y-V(C)}(w) \\
& \geqslant|X|-1+\delta(t+1)-\sum_{w \in X^{+}(T) \cup\{x\}} d_{C}(w) \\
& \geqslant|X|-1+\delta(t+1)-t-t(|X|+2) / 2 .
\end{aligned}
$$

Since the coefficient at $t$ is at least $\delta-1-(\delta+2) / 2>0$ (assuming, as we do, that $\delta>4$ ), this quantity is minimized whenever $t$ is minimized, i.e., $t=4$. We obtain $|Y| \geqslant|X|-1+5 \delta-4-2(|X|+2)$, which is minimized when $|X|=\delta$. So $|Y| \geqslant 4 \delta-9$, a contradiction.

Now suppose that for all $x \in X$ and cycles $C$ with $V(C) \cap X=X-\{x\}$, the largest $x, V(C)$-fan has size at most 3 . Choose $x \in X$ with the maximum number of neighbors of degree at least 2. If every $x \in X$ has at most 3 neighbors of degree at least 2 (and at least $\delta-3$ neighbors of degree 1$)$, then we have $|Y| \geqslant|X|(\delta-3)+3$; since $|X| \geqslant 4$, $|Y| \geqslant 4 \delta-9$, a contradiction.

Therefore $x$ has at least 4 neighbors of degree at least 2. Let $F$ be a maximum $x, V(C)$ fan of $G$ and set $T=F \cap V(C)$. By Lemma 36, $|T| \geqslant 3$, since $x$ cannot be separated from $X-x^{\prime}$ by a set of size 2 . So $|T|=3$.

By Lemma 16, $|T \cap Y| \geqslant 2$ (we apply this lemma to $G^{\prime}$, but the conclusion carries over to $G$ ). If $|T \cap Y|=3$, then since $x$ has at least 4 neighbors of degree at least 2 , there exists $y \in N(x)-V(C)$. Since all vertices in $X-\{x\}$ are contained in $C, y$ has a neighbor $x^{\prime} \in C$. But then $F \cup x y x^{\prime}$ is an $x, V(C)$-fan of size 4, a contradiction.

Finally, we may assume $T \cap V(C)=\left\{x_{1}, y_{1}, y_{2}\right\}$, where $x$ and $x_{1}$ have at least 2 common neighbors outside $C$. In particular, $\left\{y_{1}, y_{2}\right\} \subset N(x)$, and for any $x^{\prime} \neq x, x_{1}$, we have $N(x) \cap N\left(x^{\prime}\right) \subseteq\left\{y_{1}, y_{2}\right\}$, otherwise we could find a larger $x, V(C)$-fan. We will show that $N\left(y_{1}\right)=N\left(y_{2}\right)=X$. If there exists $x^{\prime}, x^{\prime \prime} \in X-\left\{x_{1}\right\}$ such that $x^{\prime} y_{i}, x^{\prime \prime} y_{i} \notin E(G)$ for some $i \in\{1,2\}$, then there cannot exist a 6 -cycle based on $\left\{x, x^{\prime}, x^{\prime \prime}\right\}$, a contradiction. 
Hence $|N(y)| \geqslant|X|-2$ which implies $N(y)=X$ by Lemma 22 (again, we apply this lemma to $G^{\prime}$, but the conclusion carries over to $G$ ).

Consider $y \in \widehat{N}(x)-\left\{y_{1}, y_{2}\right\}$. Since there is no $x, V\left(C_{x}\right)$-fan of size $4, N(y) \subseteq T \cup\{x\}$. That is, $N(y)=\left\{x, x_{1}\right\}$ and so $\widehat{N}(x) \subseteq N\left(x_{1}\right)$. Recall that we chose $x$ to have a maximum number of neighbors of degree at least 2. Additionally, note that $V(C) \cap Y \subseteq \widehat{N}(X)$. Thus $N_{C}\left(x_{1}\right)=\left\{y_{1}, y_{2}\right\}$, since otherwise $\left|\widehat{N}\left(x_{1}\right)\right|>|\widehat{N}(x)|$. But then $\left\{y_{1}, y_{2}\right\}$ separates $\left\{x, x_{1}\right\}$ from the rest of the vertices in $X$, contradicting Lemma 36 .

\section{Acknowledgement}

We thank both referees for their helpful comments.

\section{References}

[1] J. A. Bondy, Pancyclic graphs I, J. Combin. Theory, 11 (1971), 80-84.

[2] J. A. Bondy, Pancyclic graphs: recent results, Infinite and finite sets (Colloq., Keszthely, 1973; dedicated to P. Erdős on his 60th birthday), Vol. I, 181-187. Colloq. Math. Soc. János Bolyai, Vol. 10, North-Holland, Amsterdam (1975).

[3] G. A. Dirac, In abstrakten Graphen vorhandene vollstandige 4-Graphen und ihre Unterteilungen, Math. Nachr., 22 (1960), 61-85.

[4] B. Jackson, Cycles in bipartite graphs, J. Combin. Theory, Ser. B, 30 (1981), 332342.

[5] B. Jackson, Long cycles in bipartite graphs, J. Combin. Theory, Ser. B, 38 (1985), 118-131.

[6] J. Kim, personal communication.

[7] A. Kostochka, and R. Luo, On $r$-uniform hypergraphs with circumference less than r, Discrete Appl. Math. 276 (2020), 69-91.

[8] A. Kostochka, R. Luo, and D. Zirlin, Super-pancyclic hypergraphs and bipartite graphs, to appear in J. Combin. Theory, Ser. B, arXiv:1905.03758.

[9] A. Kostochka, R. Luo, M. Lavrov, and D. Zirlin, Longest cycles in 3-connected hypergraphs and bipartite graphs, submitted, arXiv:2004.08291.

[10] J. Mitchem and E. Schmeichel, Pancyclic and bipancyclic graphs - a survey, Graphs and applications (1985), 271-278.

[11] D. West, Introduction to Graph Theory, second edition, Prentice Hall, 2001. 